\title{
Editorial
}

\section{Control Design for Systems Operating in Complex Environments}

\author{
Chenguang Yang $\mathbb{D},{ }^{1}$ Zhaojie Ju, ${ }^{2}$ Xiaofeng Liu, ${ }^{3}$ Junpei Zhong $\mathbb{D},{ }^{4}$ and Andy Annamalai ${ }^{5}$ \\ ${ }^{1}$ Bristol Robotics Laboratory, University of the West of England, Bristol, UK \\ ${ }^{2}$ School of Computing, University of Portsmouth, Portsmouth, UK \\ ${ }^{3}$ College of Internet of Things Engineering, Hohai University, Changzhou, China \\ ${ }^{4}$ School of Science and Technology, Nottingham Trent University, UK \\ ${ }^{5}$ Department of Digital Futures, University of Winchester, UK \\ Correspondence should be addressed to Chenguang Yang; cyang@ieee.org
}

Received 28 May 2019; Accepted 28 May 2019; Published 12 June 2019

Copyright (C) 2019 Chenguang Yang et al. This is an open access article distributed under the Creative Commons Attribution License, which permits unrestricted use, distribution, and reproduction in any medium, provided the original work is properly cited.

In the past few decades, the technical requirements of control systems have become more stringent to be applicable to many aspects of industrial applications and automation devices, ranging from robots in manufacturing, underwater vehicles, and unmanned aerial vehicles to human-robot interaction. However, these applications and devices always need to operate in a complex environment. The growing number of applications in complex engineering environments, along with the increasing requirements and demands for the system stability, safety, and reliability, is posing new theoretical and technological challenges to the advanced controller design.

One major challenge is the provision of innovative solutions to deal with the complex operation environment. A general and illustrative definition of complex environment could be "a scenario which is dynamic and uncertain", e.g., unknown environment model, high spatiotemporal complexity, rapid changes of light intensities, and unknown disturbances. The complex and dynamic environment often leads to a rapidly increasing complexity of the control algorithm and then results in difficulties for the real-time controllers. Therefore, revealing the properties and behaviors of system operating in the complex environment could not only bring in novel control designs but also provide solutions for systems operating realistically and efficiently in complex and unmodeled scenarios.

In this special issue, we bring together works under the topic of control design for systems operating in complex environments and collect 52 papers in related fields. The contributors have investigated the design for a wide range of control systems, such as robot manipulator, aerial robots, and wheeled mobile robots and have also performed some progress on fundamental theoretical studies.

The article by S. Krafes et al. gives an updated state of the art on the control of several classes of two degrees of freedom underactuated mechanical systems such as acrobat, inverted pendulum, crane, rotational pendulum, and the inertial wheel pendulum and so on. For these underactuated systems, the authors provide a description of the mathematical models of each system and then review the classification and control strategies for these systems such as sliding mode control and intelligent control.

In the field of robot control, nine papers of the special issue investigate the control design of the robot in the complex environment with a wide range of applications, for example, the research of harvesting robot (L. Luo et al.), multirobot system (M. Liu et al.), grinding robot (S. Tang et al.), robotic manipulators (Y. Fan et al., J. Yang et al., Q. Zhang et al., and F. Zha et al.), flexible Timoshenko manipulator (J. Zhang et al.), and swarm robots (X. Lan et al.). In addition, in the field of aerial robots, $H$. Xing and Y. Yan investigate the detection of low-flying small targets in complex sea condition by proposing a complete ensemble empirical mode decomposition method. C. Huang et al. study the dynamic path planning problem of the unmanned aerial vehicles of by taking the dynamic threats and static obstacle into consideration, and the ant colony optimization and artificial potential field method is used to generate the collision-free path. Z. Chen et al. propose a finite-time 
fault-tolerant control scheme for the attitude stabilization of rigid spacecrafts.

In the field of autonomous underwater vehicle control, an observability degree analytical model is proposed by $\mathrm{H}$. Yu and $\mathrm{X}$. Zhou. To control the multiple autonomous underwater vehicles, Y. Wang and Y. Zhang investigate the coordinated depth control problem and consensus path following problem. Autonomous unmanned ground vehicles provide an excellent solution to tasks such as autonomous transportation, mining, or home service. In this research field, the control of mobile robots or wheeled ground robots has also been widely investigated; for instance, A. J. Clark et al. propose controllers for a transformable wheel mobile robot by evolving the robots' mechanical parameters and the finite state machine or the neural network. Related research topics have also been extensively investigated, such as path planning problem (P. Cui et al.), multiagent systems (X. Lan et al.), bidirectional vehicle platoon (M. Yan et al.), container loading problem (X. Xiang et al.), CNS integrated navigation system (S. Lijun et al.), collision-free control for autonomous surface vehicles (S. Gao et al.), vehicle text data compression and transmission (J. Yang et al.), and vehicle information influence degree screening (J. Yang et al.).

On the complex systems of bioinspired system and human machine interaction, S. Zhang et al. present a modeling and dynamic control of a class of semibiomimetic robotic fish. Z. Cao et al. employ the central pattern generator for path following and locomotion optimization of a snake-like robot. $\mathrm{X}$. Zhang et al. develope a state prediction model based on the extreme learning machine for a robotic fish. Y. Huang et al. develop surface electrodes with high mechanical adaptability and electrical sensitivity by presenting a self-similar inspired configuration. M. Li et al. develop a learning-based approach to transfer force-relevant skills from human demonstration to a robot. D. Wang et al. develop a control algorithm based on two microphones with nonnegative matrix factorization for the speech enhancement in complex environment. L. Zou et al. design a cascade architecture by combining a shallow $\mathrm{CNN}$ and a deep CNN to address the license plate detection problem.

About the control, optimization, and identification issues of the systems which work in complex environments, X. Yang et al. propose a sound source omnidirectional positioning calibration method based on microphone observation angle. Y. Wang et al. present a cooperative spectrum sensing method based on empirical mode decomposition and information geometry in complex electromagnetic environment. L. Fu and J. Zhao construct a novel Cartesian impedance controller based on the Maxwell model. S. Liu et al. introduce a cross field of view (FOV) real-time pose estimation system to provide high precision pose estimation of the free flight aircraft in wind tunnel environment. M. Su et al. study the angles-only guidance problem for exoatmospheric maneuvering targets by designing an integrated estimation law. J. Zhong et al. study the trajectory planning of an intermittent jumping quadruped robot with variable redundant and underactuated joints. H. Zhu et al. develop a control algorithm for uncertain building structural system by combining fuzzy adaptive control and sliding mode control. Y. Feng et al. propose a
Jiles-Atherton model to characterize the nonlinearities in the SMA-based compliant actuator.

Moreover, a number of contributions also investigate the theoretical problem of the control and modeling of systems in complex environment. H. Shi et al. propose a delayrange-dependent robust constrained model predictive control for discrete-time system with uncertainties and unknown disturbances. L.-N. Zhu et al. introduce a novel manufacturing scheme, MBSPHE-CSCCM, for the multibatch subtasks parallel-hybrid execution cloud service in could manufacturing. $\mathrm{H}$. Yang et al. introduce a single parameter adaptive neural network control method for unknown nonlinear systems with bounded external disturbances. M. Xie et al. proposed a strong local diagnosability for a hypercube network. Y.-S. Wei and Q.-Y. Xu present a proportional type iterative learning control (ILC) law for linear discrete-time systems with randomly variable input trail length. R. Wang et al. present a theoretical model to analyze the performance degradation of control systems subject to common-source digital upsets. X. Lv et al. investigate the synchronization problem for neural networks by using feedback control. C. Ren and S. He proposed an observer-based finite-time control algorithm for a class of Markov jump system in a complex environment. Y. Peng et al. develop a periodically intermittent controller for a class of coupled hybrid stochastic delayed bidirectional associative memory neural networks. G. Peng et al. propose an adaptive Monte Carlo Localization algorithm to build a laser and radar based robot localization system in a complex and unstructured environment. Z. Zhao et al. develop a vibration boundary control or an axial string system subject to input restriction and external disturbance.

The selected papers in the special issue could not cover all the recent advances of the control design for systems operating in complex environments, yet they present the state-of-the-art progress in this area. We hope these valuable papers enrich knowledge in the complex systems community and provide guidance to the readers who are interested in this topic.

\section{Conflicts of Interest}

The editors declare that they have no conflicts of interest regarding the publication of this special issue.

\section{Acknowledgments}

The guest editorial team would like to express their appreciations to all the authors for their interest in selecting this special issue and also wish to thank the anonymous reviewers for their careful reading and many insightful comments and suggestions.

Chenguang Yang
Zhaojie Ju
Xiaofeng Liu
Junpei Zhong
Andy Annamalai 


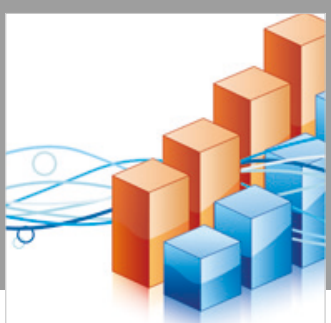

Advances in

Operations Research

\section{-n-m}
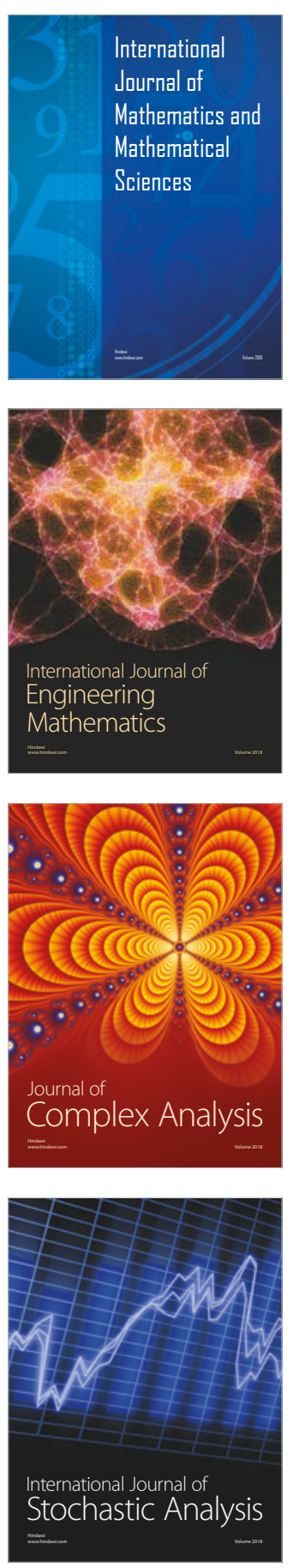
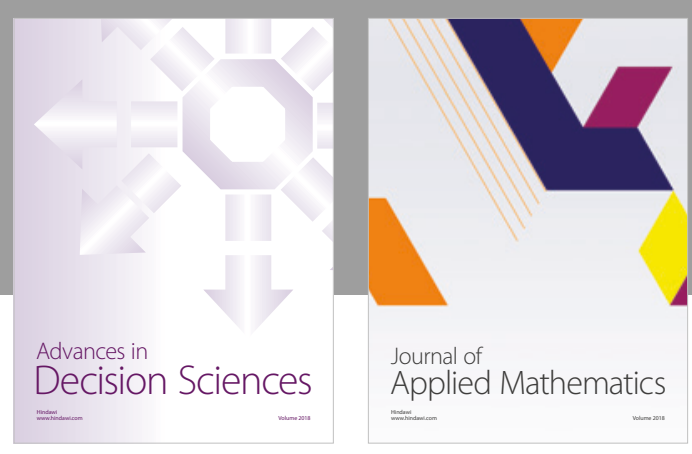

Journal of

Applied Mathematics
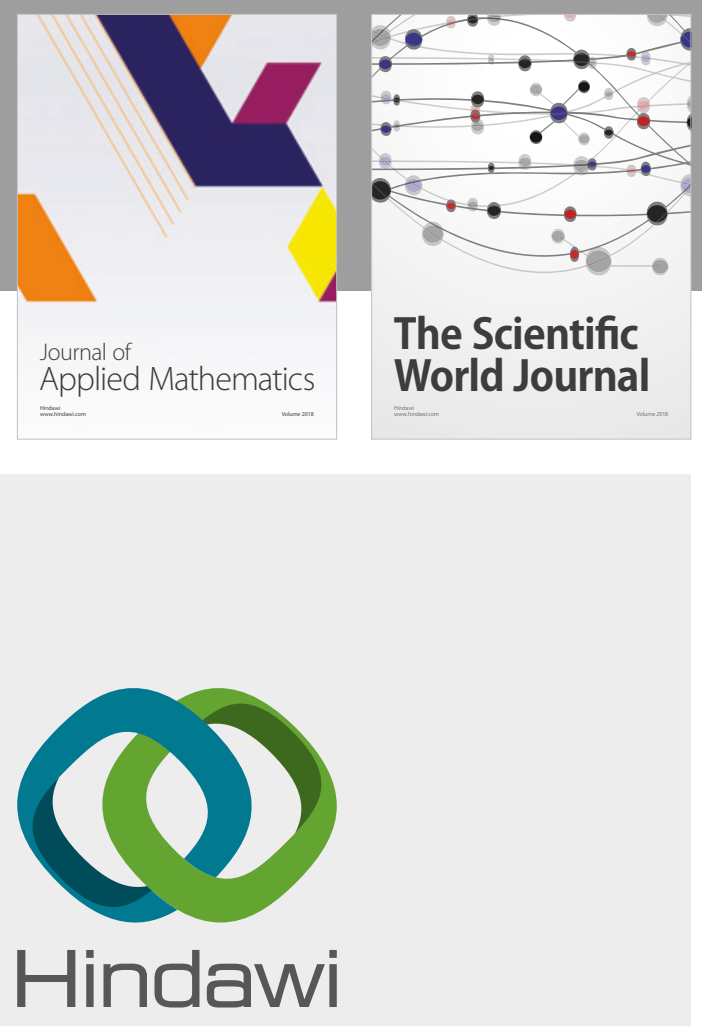

Submit your manuscripts at

www.hindawi.com

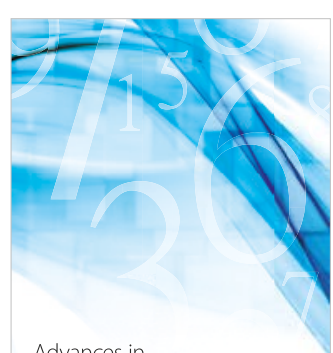

Advances in
Numerical Analysis
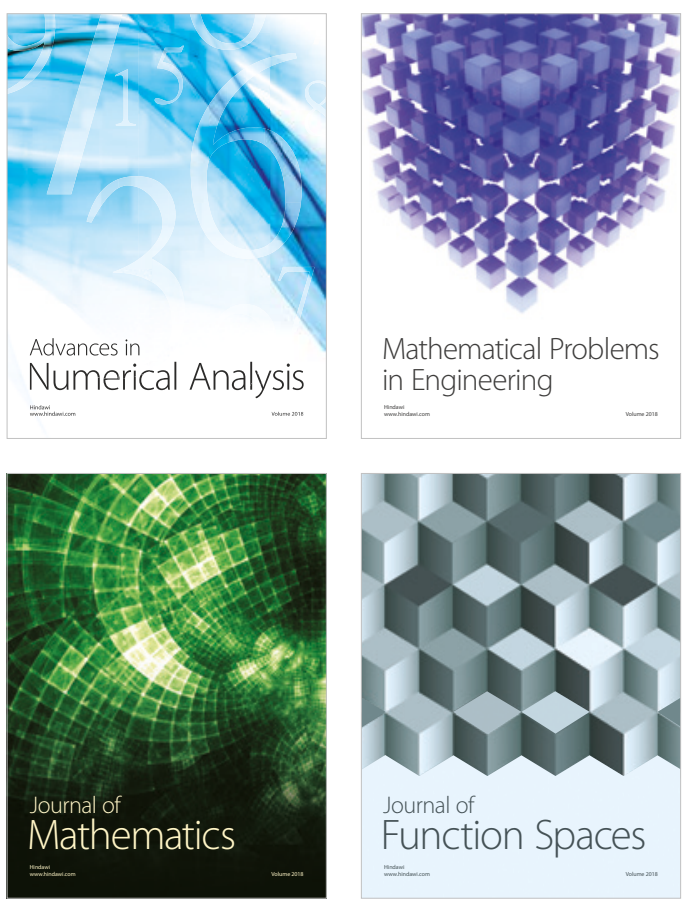

Mathematical Problems in Engineering

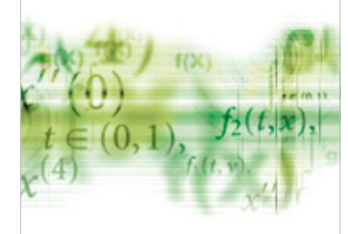

International Journal of

Differential Equations

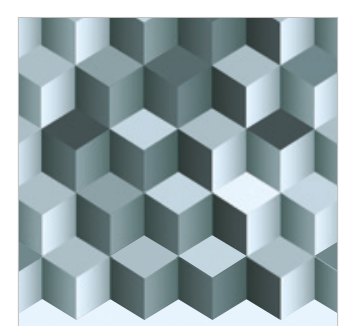

Journal of

Function Spaces
The Scientific

World Journal

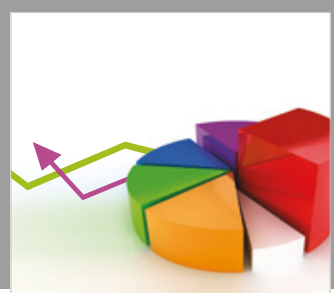

Journal of

Probability and Statistics
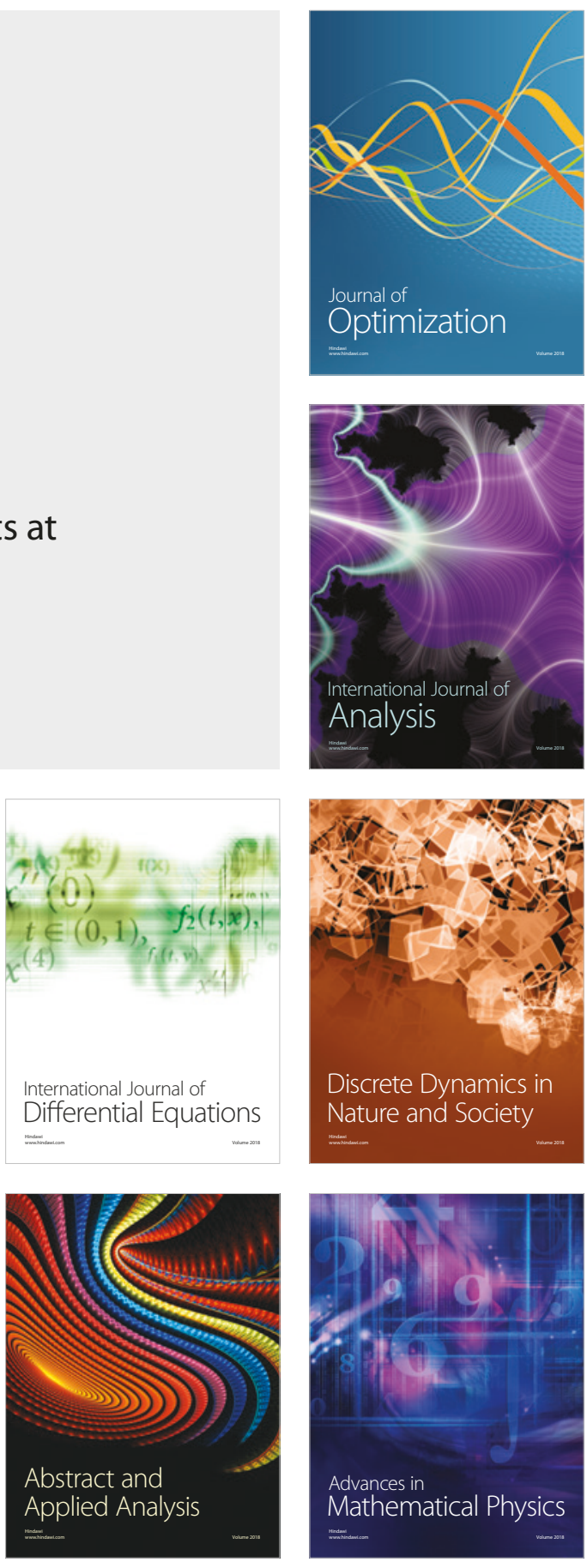